\title{
The role of the comprehensive complication index for the prediction of survival after liver transplantation
}

\author{
Quirino Lai ${ }^{1}$ (D) Fabio Melandro ${ }^{1} \cdot$ Greg Nowak $^{2}$ - Daniele Nicolini ${ }^{3} \cdot$ Samuele lesari ${ }^{4,5} \cdot$ Elisa Fasolo $^{6}$. \\ Gianluca Mennini ${ }^{1}$. Antonio Romano ${ }^{2}$. Federico Mocchegiani ${ }^{3} \cdot$ Kevin Ackenine $^{4} \cdot$ Marina Polacco $^{6}$. \\ Laura Marinelli ${ }^{3}$. Olga Ciccarelli ${ }^{4}$ - Giacomo Zanus ${ }^{6} \cdot$ Marco Vivarelli $^{3} \cdot$ Umberto Cillo $^{6} \cdot$ Massimo Rossi $^{1}$. \\ Bo-Göran Ericzon ${ }^{2} \cdot$ Jan Lerut ${ }^{4}$
}

Received: 21 April 2020 / Accepted: 30 August 2020 / Published online: 6 September 2020

(c) The Author(s) 2020

\begin{abstract}
In the last years, several scoring systems based on pre- and post-transplant parameters have been developed to predict early post-LT graft function. However, some of them showed poor diagnostic abilities. This study aims to evaluate the role of the comprehensive complication index (CCI) as a useful scoring system for accurately predicting 90-day and 1-year graft loss after liver transplantation. A training set $(n=1262)$ and a validation set $(n=520)$ were obtained. The study was registered at https://www.ClinicalTrials.gov (ID: NCT03723317). CCI exhibited the best diagnostic performance for 90 days in the training $(\mathrm{AUC}=0.94 ; p<0.001)$ and Validation Sets $(\mathrm{AUC}=0.77 ; p<0.001)$ when compared to the BAR, D-MELD, MELD, and EAD scores. The cut-off value of 47.3 (third quartile) showed a diagnostic odds ratio of 48.3 and 7.0 in the two sets, respectively. As for 1-year graft loss, $\mathrm{CCI}$ showed good performances in the training (AUC $=0.88 ; p<0.001$ ) and validation sets (AUC $=0.75 ; p<0.001)$. The threshold of 47.3 showed a diagnostic odds ratio of 21.0 and 5.4 in the two sets, respectively. All the other tested scores always showed AUCs $<0.70$ in both the sets. CCI showed a good stratification ability in terms of graft loss rates in both the sets $(\log$-rank $p<0.001)$. In the patients exceeding the CCI ninth decile, 1 -year graft survival rates were only $0.7 \%$ and $23.1 \%$ in training and validation sets, respectively. CCI shows a very good diagnostic power for 90 -day and 1-year graft loss in different sets of patients, indicating better accuracy with respect to other pre- and post-LT scores. Clinical Trial Notification: NCT03723317.
\end{abstract}

Keywords MELD $\cdot$ Retransplantation $\cdot$ Graft survival $\cdot$ Survival prediction $\cdot$ Allograft dysfunction

\section{Abbreviations}

AUC Area under the curve

BAR Balance of risk

CCI Comprehensive complication index

CI Confidence intervals

DBD Deceased-brain donor

DCD Deceased-cardiac donor
DOR Diagnostic odds ratio

EAD Early allograft dysfunction

HR Hazard ratio

IQR Interquartile ranges

LDLT Living donor

LT Liver transplantation

MELD Model for end-stage liver disease
Quirino Lai

lai.quirino@libero.it

1 General Surgery and Organ Transplantation Unit, Department of Surgery, Sapienza University of Rome, Umberto I Polyclinic of Rome, Viale del Policlinico 155, 00161 Rome, Italy

2 Division of Transplantation Surgery, Karolinska University Hospital Huddinge, Solna, Sweden

3 Unit of Hepatobiliary Surgery and Transplantation, Polytechnic University of Marche, Azienda
Ospedaliero-Universitaria “Ospedali Riuniti” Torrette, Ancona, Italy

4 Starzl Unit of Abdominal Transplantation, Pôle de Chirurgie Expérimentale et Transplantation, Institut de Recherche Expérimentale et Clinique, Université Catholique de Louvain, Brussels, Belgium

5 Department of Biotechnological and Applied Clinical Sciences, University of L'Aquila, L’Aquila, Italy

6 Department of Surgery, Oncology and Gastroenterology, University of Padua, Padua, Italy 


\section{MOF Multiorgan failure \\ PNF Primary non-function \\ RRT Renal replacement therapy \\ SOFT Survival outcomes following liver transplantation}

\section{Introduction}

In the last years, several scoring systems have been developed with the intent to predict early clinical course after liver transplantation (LT). The model for end-stage liver disease (MELD) is recognized as the most accurate liver allograft allocation model, and it prioritizes patients according to the severity of their disease $[1,2]$. However, several studies have shown that MELD alone fails to predict early post-transplant survival rates [3, 4]. Consequently, other scoring systems based on pre- or post-transplant available variables have been developed to identify cases with a high risk for transplant failure. Among them, the "pre-transplant" scores D-MELD and balance of risk (BAR) [5, 6], and the "post-transplant" score early allograft dysfunction (EAD) [7] proved to predict post-transplant survival satisfactorily.

Recently, the comprehensive complication index (CCI) has been developed to assess the actual complication rate after surgery [8]. Some reports have shown excellent prognostic power of this score in different fields [9-11]. No study to date has investigated the role of CCI in 90-day and 1-year prognostication of graft loss after LT.

This study aims to compare the abilities of CCI vs. other commonly adopted pre- and post-transplant scoring systems in diagnosing 90-day and 1-year post-transplant liver graft loss. The diagnostic capabilities were investigated in a training set and validated in a validation set.

\section{Materials and methods}

Training set was generated retrospectively analyzing 1262 patients undergoing a first LT during the period January 1, 2005-December 31, 2016. Four European collaborative LT Centres were involved in creating the Training Set, namely the Polytechnic University of Marche, Ancona (Italy), Université Catholique de Louvain, Brussels (Belgium), Sapienza University, Rome (Italy), and University of Padua (Italy). Exclusion criteria for patient selection were: (a) living donation, (b) combined transplant, (c) domino transplant, and (d), pediatric $(<18$ years $)$ transplant.

Validation Set was created retrospectively analyzing the data of 520 patients transplanted during the same timeframe in the Karolinska Institute of Stockholm (Sweden). The same exclusion criteria were adopted. The study was registered at https://www.ClinicalTrials.gov (ID: NCT03723317).

\section{Definitions}

Organ procurement was defined as "local" when done in the same region in which the LT was performed. All complications were graded according to the Clavien-Dindo Classification [12]. A web-calculator was used for estimating BAR and CCI (available at https://www.assessurge ry.com/).

The CCI was calculated using the following original algorithm: $\mathrm{CCI}=[\sqrt{ }(\mathrm{wC} 1+\mathrm{wC} 2 \ldots+\mathrm{wC} x)] / 2$.

The CCI is based on the complication grading by Clavien-Dindo Classification and implements every occurred weighted complication (wC) after an intervention. Clavien-Dindo grade I corresponds to 8.7, grade II to 20.9, grade IIIa to 26.2, grade IIIb to 33.7 , grade IVa to 42.4 , grade IVb to 46.2 , and grade V to 100 . All the complications collected were summed, even if the same patient received several times multiple administrations of the same medical (i.e., blood transfusion) or interventional (i.e., various radiological or surgical approaches) treatment. The overall morbidity is reflected on a scale from 0 (no complication) to 100 (death).

Retransplantation during the first hospitalization was calculated as IVa (liver failure) plus IIIb (reoperation) complication. Multiorgan failure (MOF) was defined as the presence of at least two organ failures and ranked as grade IVb complication. Primary non-function (PNF) was identified as a liver failure observed for non-technical reasons within seven days after surgery and ranked as IVa complication.

EAD was defined according to the Olthoff criteria [7], and classified as grade II complication. Mild renal dysfunction was associated with a serum creatinine increase overpassing the threshold of $1.5 \mathrm{mg} / \mathrm{dL}$ but not requiring renal replacement therapy (RRT), and corresponded to a grade I complication. In the case of RRT, a grade IVa complication was defined. Myelotoxicity was defined as the presence of at least one of the following conditions: anemia (hemoglobin $<8 \mathrm{~g} / \mathrm{dL}$ ) in the absence of bleeding, leukopenia $(<3500 / \mu \mathrm{L})$, or severe thrombocytopenia $(<30,000 / \mu \mathrm{L})$, being classified as a grade I complication.

\section{Statistical analysis}

Continuous variables were reported as medians and interquartile ranges (IQR). Categorical variables were reported as numbers and percentages. Missing data always involved $<10 \%$ per variable and were handled using the maximum likelihood estimation method. Mann-Whitney $U$ test was used for comparisons between groups in case of continuous variables, and Fisher's exact test was adopted in case of categorical variables. 
A univariate Cox regression analysis was performed in the training set for the identification of the risk factors for graft loss. All the variables with a $p$ value $<0.20$ were introduced into a multivariable model. A multivariable Cox regression model was constructed adopting the backward conditional method [13]. Beta-coefficients, standard errors, the hazard ratio (HR), and $95 \%$ confidence intervals (95\% CI) were reported.

$\mathrm{C}$-statistics was used for comparing the diagnostic ability of different scores in terms of 90-day and 1-year graft loss in both the training and validation set. Specifically, CCI was compared with MELD, D-MELD, BAR, and EAD. Areas under the curve (AUC), standard errors, and $95 \%$ CI were reported. The following CCI cut-off values were investigated in the training set: first quartile, median, third quartile, and ninth decile. Sensitivity, specificity, and diagnostic odds ratio (DOR) were reported for each cut-off value. The higher the DOR value, the greater its discriminative power. The same CCI threshold values obtained in the training set were validated in the Validation Set. Graft survival rates were estimated with the Kaplan-Meier method; the log-rank test was used for evaluating survival differences. A $p$ value $<0.05$ was considered statistically significant in all analyses. Statistical reports and plots were performed using the SPSS statistical package version 24.0 (SPSS Inc., Chicago, IL, USA).

\section{Results}

The training and validation sets were composed of 1262 and 520 LT recipients. All grafts were procured from donors after brain death.

In the training set, the median follow-up was 3.7 years (IQR 1.1-7.6), with 1108 (87.8\%) and 991 (78.5\%) cases exceeding 90 days and 1 year, respectively.

During the entire study period, 371 (29.4\%) patients died: of whom 154 (12.8\%) and 66 (5.2\%) within 90 days and during the time interval of 91-365 days, respectively.

Four hundred and nine (32.4\%) grafts were lost: 186 (14.7\%) and 70 (5.5\%) within 90 days and 91-365 days, respectively. Seventy-eight $(6.2 \%)$ retransplantations were performed: $54(4.5 \%)$ and $12(1.0 \%)$ within 90 days and 91-365 days, respectively.

In the validation set, the median follow-up was 4.8 years (IQR 3.0-7.2), with 504 (97.9\%) and 485 (93.3\%) cases exceeding 90 days and 1 year, respectively.

During the entire study period, $104(20.0 \%)$ patients died: $15(2.9 \%)$ within 90 days and $18(3.5 \%)$ during the time interval of 91-365 days from LT.

One hundred and fourteen (21.9\%) grafts were lost: 17 (3.3\%) within 90 days and 20 (3.8\%) during 91-365 days. Twelve (2.3\%) retransplantations were performed: one $(0.2 \%)$ within 90 days and three $(0.6 \%)$ during $91-365$ days.

\section{Baseline characteristics}

The characteristics of the sets are displayed in Table 1.

In the training set, median lab-MELD was 15 points, with $161(12.8 \%)$ patients showing a MELD $\geq 30$. Median waiting time and age at LT were 4 months and 56 years, respectively. HCC was the main indication for LT in 527 (41.8\%) patients. The main cause of the liver disease was HCV-related cirrhosis (35.7\%). Median donor age was 57 years, with 328 (26.0\%) and 77 (6.1\%) donors exceeding 70 and 80 years. The leading brain-death cause was cerebrovascular accident $(n=785 ; 62.2 \%)$. In approximately half of the cases, the procurement was performed in a local hospital. Median cold and warm ischemia times were $7.2 \mathrm{~h}$ and $45 \mathrm{~min}$. Median BAR score was 5; $77(6.1 \%)$ and six $(0.5 \%)$ recipients had a score exceeding 15 and 20, respectively.

In the validation set, median lab-MELD was 25 points, with $118(22.7 \%)$ recipients presenting a MELD $\geq 30$. Median waiting time and age at LT were 2 months and 54 years, respectively. HCC was the leading indication for LT in $131(25.2 \%)$ patients. HCV-related cirrhosis was reported in 148 (28.5\%) cases. Pathologies uncommonly reported in the Training Set were, on the opposite, commonly reported in the validation set: biliary pathologies like primary biliary cholangitis and primary sclerosing cholangitis were reported in 124/520 (23.8\%) cases, followed by $37(7.1 \%)$ cases of familiar amyloid polyneuropathy, and 25 (4.8\%) cases of autoimmune hepatitis.

Median donor age was 57 years, with 91 (17.5\%) and eight (1.5\%) donors exceeding 70 and 80 years. The leading cause of brain death was a cerebrovascular accident $(n=346$; $66.5 \%)$. In $\sim 75 \%$ of cases, the procurement was performed in a local hospital. Median cold and warm ischemia times were $8.3 \mathrm{~h}$ and $40 \mathrm{~min}$. Median BAR score was $11 ; 30$ (5.8\%) recipients had a score exceeding 15 , while no case exceed 20.

\section{Post-transplant course}

The postoperative courses of the two sets are displayed in Table 2. In the training set, median intensive care stay and overall length of hospital stay were four and 17 days. According to the highest Clavien-Dindo grade, 182 (14.4\%) patients had no complications, $184(14.6 \%)$ and 406 (32.2\%) had grades I and II, $79(6.2 \%)$ and $146(11.5 \%)$ had grades IIIa and IIIb, $96(7.7 \%)$ and $21(1.7 \%)$ had grades IVa and $\mathrm{IVb}$, and $148(11.7 \%)$ died (grade V). One hundred and fifty-eight (12.5\%) patients required a "IIIa procedure". The most common procedures were thoracic $(n=54 ; 4.3 \%)$ or abdominal drainage $(n=45 ; 3.6 \%)$. Two hundred and seventy-one $(21.5 \%)$ patients required a "IIIb procedure". The most common procedure was reoperation for bleeding $(n=88 ; 7.0 \%)$, followed by explorative laparotomy $(n=73$; 
Table 1 Characteristics of recipients, donors, and transplants in the training and validation sets

\begin{tabular}{|c|c|c|c|}
\hline \multirow[t]{2}{*}{ Variables } & \multicolumn{2}{|l|}{ Median (IQR) or $n(\%)$} & \multirow[t]{2}{*}{$p$ value } \\
\hline & Training set $(N=1262)$ & Validation set $(N=520)$ & \\
\hline \multicolumn{4}{|l|}{ Recipient } \\
\hline Age at LT (years) & $56(49-62)$ & $54(44-62)$ & 0.001 \\
\hline Male gender & $949(75.2)$ & $355(68.3)$ & 0.003 \\
\hline Waiting time (months) & $4(1-10)$ & $2(1-5)$ & 0.003 \\
\hline MELD la & $15(10-22)$ & $26(23-29)$ & $<0.001$ \\
\hline \multicolumn{4}{|l|}{ Disease } \\
\hline $\mathrm{HCC}$ & $527(41.8)$ & $131(25.2)$ & $<0.001$ \\
\hline $\mathrm{HCV}$ & $451(35.7)$ & $148(28.5)$ & 0.003 \\
\hline $\mathrm{HBV}$ & $174(13.8)$ & $35(6.7)$ & $<0.001$ \\
\hline Alcohol & $433(34.3)$ & $109(21.0)$ & $<0.001$ \\
\hline Acute liver failure & $64(5.1)$ & $20(3.8)$ & 0.3 \\
\hline NASH & $88(7.0)$ & $47(9.0)$ & 0.1 \\
\hline Other & $223(17.7)$ & $235(45.2)$ & $<0.001$ \\
\hline \multicolumn{4}{|l|}{ Donor } \\
\hline Age (years) & $57(43-70)$ & $57(44-67)$ & 0.2 \\
\hline Male gender & $719(57.0)$ & $292(56.2)$ & 0.8 \\
\hline BMI $\left(\mathrm{kg} / \mathrm{m}^{2}\right)$ & $25(23-28)$ & $25(22-28)$ & 0.06 \\
\hline ICU stay (days) & $3(2-5)$ & $2(1-3)$ & $<0.001$ \\
\hline \multicolumn{4}{|l|}{ Cause of death } \\
\hline Trauma & $337(26.7)$ & $76(14.6)$ & $<0.001$ \\
\hline Anoxia & $94(7.4)$ & $92(17.7)$ & $<0.001$ \\
\hline CVA & $785(62.2)$ & $346(66.5)$ & 0.09 \\
\hline Other & $62(4.9)$ & $5(1.0)$ & $<0.001$ \\
\hline Cardiac arrest & $158(12.5)$ & $133(25.6)$ & $<0.001$ \\
\hline History of DM & $90(7.1)$ & $39(7.5)$ & 0.8 \\
\hline Local procurement & $640(50.7)$ & $387(74.4)$ & $<0.001$ \\
\hline \multicolumn{4}{|l|}{ Transplant } \\
\hline Cold ischemia time (min) & $434(360-533)$ & $496(411-568)$ & $<0.001$ \\
\hline Warm ischemia time (min) & $45(35-60)$ & $40(34-50)$ & $<0.001$ \\
\hline D-MELD & 815 (499-1245) & 1454 (1084-1826) & $<0.001$ \\
\hline BAR & $5(3-9)$ & $11(8-13)$ & $<0.001$ \\
\hline \multicolumn{4}{|l|}{ Post-LT clinical course } \\
\hline ICU stay (days) & $4(2-7)$ & $1(1-2)$ & $<0.001$ \\
\hline Total length of stay (days) & $17(13-27)$ & $14(11-20)$ & $<0.001$ \\
\hline Total bilirubin seventh day $(\mathrm{mg} / \mathrm{dL})$ & $4.5(1.8-8.3)$ & $2.1(1.0-4.2)$ & $<0.001$ \\
\hline INR 7th day & $1.00(1.00-1.16)$ & $1.20(1.10-1.40)$ & $<0.001$ \\
\hline ALT peak during first week (IU/L) & $745(382-1509)$ & $364(202-707)$ & $<0.001$ \\
\hline AST peak during first week (IU/L) & 907 (447-2086) & $252(143-447)$ & $<0.001$ \\
\hline
\end{tabular}

$L T$ liver transplantation, IQR interquartile ranges, MELD model for end-stage liver disease, $H C C$ hepatocellular cancer, $H C V$ hepatitis $\mathrm{C}$ virus, $H B V$ hepatitis B virus, $N A S H$ non-alcoholic steatohepatitis, $B M I$ body mass index, $I C U$ intensive care unit, $C V A$ cerebrovascular accident, $D M$ diabetes mellitus, $D-M E L D$ donor-MELD, BAR balance of risk, INR international normalized ratio, $A L T$ alanine aminotransferase, $A S T$ aspartate aminotransferase
$5.8 \%)$. Retransplantation during the same hospital stay of the first transplant was necessary for 45 (3.6\%) recipients. MOF was observed in $45(3.6 \%)$ recipients. EAD and PNF were reported in $508(40.3 \%)$ and $37(2.9 \%)$ cases. Mild renal dysfunction and RRT were detected in $90(7.1 \%)$ and $91(7.2 \%)$ patients. Vascular and biliary complications were reported in $80(6.3 \%)$ and $110(8.7 \%)$ subjects. Median CCI value was 29.3 (IQR: 12.2-47.6): CCI values <20, 20-39, 40-49, 50-99, and 100 were observed in 366 (29.0\%), 483 (38.3\%), 121 (9.6\%), 144 (11.4\%), and 148 (11.7\%) patients.

In the validation set, median intensive care stay and overall length of hospital stay were one and 14 days. According to the highest Clavien-Dindo grade observed, $148(28.5 \%)$ and $152(29.2 \%)$ patients had a grade I and II, 77 (14.8\%) 
Table 2 Interventional procedures performed and complications reported in the training and validation sets

\begin{tabular}{|c|c|c|c|}
\hline Variables & $\begin{array}{l}\text { Training set }(N=1262) \\
\text { Median (IQR) and } n(\%)\end{array}$ & Validation set $(N=520)$ & $p$ value \\
\hline CD score IIIa & $158(12.5)$ & $134(25.8)$ & $<0.001$ \\
\hline Biliary stenting & $34(2.7)$ & $29(5.6)$ & 0.004 \\
\hline HA stenting & $10(0.8)$ & $1(0.2)$ & 0.2 \\
\hline Abdominal drainage & $45(3.6)$ & $38(7.3)$ & 0.001 \\
\hline Thoracic drainage & $54(4.3)$ & $55(10.6)$ & $<0.001$ \\
\hline Arterial embolization & $17(1.3)$ & $0(-)$ & 0.005 \\
\hline Other & $28(2.2)$ & $42(8.1)$ & $<0.001$ \\
\hline CD score IIIb & $271(21.5)$ & $101(19.4)$ & 0.4 \\
\hline Bleeding control & $88(7.0)$ & $41(7.9)$ & 0.5 \\
\hline Immediate retransplantation & $45(3.6)$ & $3(0.6)$ & $<0.001$ \\
\hline Biliary redo & $61(4.8)$ & $9(1.7)$ & 0.002 \\
\hline $\mathrm{HA} / \mathrm{PV}$ redo & $36(2.9)$ & $2(0.4)$ & $<0.001$ \\
\hline Explorative laparotomy & $73(5.8)$ & $33(6.3)$ & 0.7 \\
\hline Tracheotomy & $20(1.6)$ & $11(2.1)$ & 0.4 \\
\hline Depacking & $14(1.1)$ & $1(0.2)$ & 0.08 \\
\hline Two-time closure & $6(0.5)$ & $5(1.0)$ & 0.3 \\
\hline Other & $27(2.1)$ & $26(5.0)$ & $<0.001$ \\
\hline MOF & $45(3.6)$ & $12(2.3)$ & 0.2 \\
\hline PNF & $37(2.9)$ & $3(0.6)$ & 0.001 \\
\hline Cardiac failure/ischemic & $32(2.5)$ & $10(1.9)$ & 0.5 \\
\hline HD replacement & $91(7.2)$ & $39(7.5)$ & 0.8 \\
\hline Respiratory failure & $39(3.1)$ & $31(6.0)$ & 0.007 \\
\hline EAD & $508(40.3)$ & $87(16.7)$ & $<0.001$ \\
\hline Acute rejection & $211(16.7)$ & $95(18.3)$ & 0.4 \\
\hline HAT & $38(3.0)$ & $1(0.2)$ & $<0.001$ \\
\hline HA stenosis & $22(1.7)$ & $0(-)$ & 0.001 \\
\hline PV thrombosis/stenosis & $20(1.6)$ & $1(0.2)$ & 0.01 \\
\hline Biliary stenosis & $48(3.8)$ & $10(1.9)$ & 0.04 \\
\hline Biliary fistula & $62(4.9)$ & $1(0.2)$ & $<0.001$ \\
\hline Abdominal bleeding & $108(8.6)$ & $3(0.6)$ & $<0.001$ \\
\hline Infection & $438(34.7)$ & $188(36.2)$ & 0.6 \\
\hline Neurological/psychiatric & $202(16.0)$ & $98(18.8)$ & 0.2 \\
\hline Cardiac electric & $32(2.5)$ & $29(5.6)$ & 0.002 \\
\hline Mild acute renal dysfunction & $90(7.1)$ & $19(3.7)$ & 0.005 \\
\hline Ascites & $225(17.8)$ & $49(9.4)$ & $<0.001$ \\
\hline Diarrhoea & $23(1.8)$ & $25(4.8)$ & 0.001 \\
\hline Gastrointestinal bleeding & $21(1.7)$ & $28(5.4)$ & $<0.001$ \\
\hline Myelotoxicity & $149(11.8)$ & $40(7.7)$ & 0.01 \\
\hline Intestinal occlusion/peritonitis & $21(1.7)$ & $3(0.6)$ & 0.07 \\
\hline Pneumothorax & $11(0.9)$ & $1(0.2)$ & 0.2 \\
\hline CCI & $29.3(12.2-47.6)$ & $24.2(8.7-44.4)$ & 0.3 \\
\hline
\end{tabular}

$I Q R$ interquartile ranges, $C D$ Clavien-Dindo, $H A$ hepatic artery, $L T$ liver transplantation, $P V$ portal vein, $M O F$ multiorgan failure, $P N F$ primary non-function, $H D$ hemodialysis, $E A D$ early allograft dysfunction, $H A T$ hepatic artery thrombosis, $C C I$ comprehensive complication index and $75(14.4 \%)$ had grades IIIa and IIIb, $46(8.8 \%)$ and 13 (2.5\%) had grades IVa and IVb, and, lastly, nine (1.7\%) had a grade $\mathrm{V}$ complication. A total of 134 (25.8\%) patients required a "IIIa procedure". The most common procedures were thoracic $(n=55 ; 10.6 \%)$ or abdominal drainage $(n=38$;
7.3\%). Two hundred and one (19.4\%) patients required a "IIIb procedure". The most common was reoperation for bleeding ( $n=41 ; 7.9 \%$ ), followed by explorative laparotomy $(n=33 ; 6.3 \%)$. Early retransplantation was necessary in only three $(0.6 \%)$ cases. MOF was diagnosed in $12(2.3 \%)$ 
recipients. EAD and PNF were observed in 87 (16.7\%) and three $(0.6 \%)$ cases. Mild renal dysfunction and RRT were reported in $19(3.7 \%)$ and $39(7.5 \%)$ patients. Vascular and biliary complications were reported in two $(0.4 \%)$ and $11(2.1 \%)$ subjects. Median CCI value was 24.2 (IQR: 8.7-44.4): CCI values <20, 20-39, 40-49, 50-99, and 100 were observed in 148 (28.5\%), 220 (42.3\%), 57 (11.0\%), 86 $(16.5 \%)$, and nine (1.7) patients.

\section{Risk factors for overall risk of graft loss}

Eighteen different covariates identifiable before or during the post-LT hospital stay were tested in the training set. First, a univariate Cox regression analysis was displayed with the intent to identify the risk factors for graft loss. After selecting only the statistically significant variables, and removing the possible causes of co-linearity, a multivariable Cox regression model was built. Three independent risk factors for graft loss were identified: donor age $(\mathrm{HR}=1.01 ; p$ value $=0.002)$, BAR score $(\mathrm{HR}=1.03 ; p$ value $=0.01)$ and
CCI $(\mathrm{HR}=1.05 ; p$ value $<0.001)$ (Table 3$)$. Interestingly, CCI presented a very high Wald value (552.95) with respect to donor age and BAR (9.38 and 6.58, respectively), thus showing a high contribution of this individual predictor in the construction of the given model.

\section{0-Day graft loss diagnostic ability}

The diagnostic ability of five different scoring systems was evaluated in both the sets, with the intent to identify the best diagnostic test for 90-day graft loss (Table 4).

CCI exhibited the best diagnostic performances in both Training (AUC $=0.94,95 \% \mathrm{CI}=0.92-0.96 ; p<0.001)$ and Validation Sets (AUC $=0.77,95 \%$ CI $=0.62-0.93 ; p<0.001$ ) when compared to the BAR, D-MELD, MELD, and EAD scores. All the other scores always showed inferior AUCs, only ranging $0.58-0.60$ and $0.47-0.57$, respectively.

In the training set, the CCI cut-off value corresponding to the first quartile (12.2 points) yielded a sensitivity of 98.4 and a specificity of $27.7(\mathrm{DOR}=23.6)$. The threshold value

Table 3 Univariable and multivariable Cox regression analyses for the overall risk of graft loss after LT in the training set

\begin{tabular}{|c|c|c|c|c|c|c|c|c|c|}
\hline \multirow[t]{3}{*}{ Variables } & \multicolumn{9}{|l|}{ Training set $(N=1262)$} \\
\hline & \multicolumn{4}{|l|}{ Univariable analysis } & \multicolumn{5}{|l|}{ Multivariable analysis } \\
\hline & Beta-coefficient \pm SE & OR & $95 \% \mathrm{CI}$ & $p$ value & Beta-coefficient \pm SE & Wald & HR & $95 \% \mathrm{CI}$ & $p$ value \\
\hline Waiting time (per day) & $0.00 \pm 0.00$ & 1.00 & $1.00-1.00$ & 0.7 & - & - & - & - & - \\
\hline Patient age at LT (per year) & $-0.00 \pm 0.01$ & 1.00 & $0.99-1.01$ & 0.8 & - & - & - & - & - \\
\hline Patient male gender (yes vs. no) & $0.11 \pm 0.12$ & 1.11 & $0.89-1.40$ & 0.4 & - & - & - & - & - \\
\hline Patient BMI at LT (per unit) ${ }^{\mathrm{a}}$ & $-0.02 \pm 0.01$ & 0.98 & $0.96-1.00$ & 0.08 & - & - & - & - & - \\
\hline MELD at LT (per point) ${ }^{\mathrm{b}}$ & $0.02 \pm 0.01$ & 1.02 & $1.01-1.03$ & 0.002 & - & - & - & - & - \\
\hline Donor age $\left(\right.$ per year) ${ }^{\mathrm{a}}$ & $0.01 \pm 0.00$ & 1.01 & $1.00-1.02$ & 0.001 & $0.01 \pm 0.00$ & 9.38 & 1.01 & $1.00-1.01$ & 0.002 \\
\hline Donor male gender (yes vs. no) & $-0.06 \pm 0.10$ & 0.94 & $0.77-1.14$ & 0.5 & - & - & - & - & - \\
\hline Donor BMI at LT (per unit) & $0.01 \pm 0.01$ & 1.01 & $0.98-1.03$ & 0.5 & - & - & - & - & - \\
\hline CVA as cause of donor death ${ }^{\mathrm{a}}$ & $0.26 \pm 0.10$ & 1.29 & $1.05-1.58$ & 0.01 & - & - & - & - & - \\
\hline Donor ICU stay (per day) & $0.00 \pm 0.01$ & 1.00 & $0.98-1.03$ & 0.9 & - & - & - & - & - \\
\hline Donor DM2 ${ }^{\mathrm{a}}$ & $0.26 \pm 0.18$ & 1.30 & $0.91-1.85$ & 0.2 & - & - & - & - & - \\
\hline Donor local procurement & $-0.09 \pm 0.10$ & 0.91 & $0.75-1.11$ & 0.4 & - & - & - & - & - \\
\hline CIT (per min) & $0.00 \pm 0.00$ & 1.00 & $1.00-1.00$ & 0.5 & - & - & - & - & - \\
\hline WIT $(\text { per min) })^{\mathrm{a}}$ & $0.01 \pm 0.00$ & 1.01 & $1.00-1.01$ & 0.003 & - & - & - & - & - \\
\hline BAR score (per point) ${ }^{\mathrm{a}}$ & $0.04 \pm 0.01$ & 1.04 & $1.01-1.06$ & 0.002 & $0.03 \pm 0.01$ & 6.58 & 1.03 & $1.01-1.05$ & 0.01 \\
\hline D-MELD (per 100 points) ${ }^{a, b}$ & $0.03 \pm 0.01$ & 1.03 & $1.02-1.05$ & $<0.001$ & - & - & - & - & - \\
\hline $\mathrm{EAD}^{\mathrm{a}}$ & $0.44 \pm 0.10$ & 1.55 & $1.28-1.89$ & $<0.001$ & - & - & - & - & - \\
\hline $\mathrm{CCI}^{\mathrm{a}}$ & $0.04 \pm 0.00$ & 1.04 & $1.04-1.05$ & $<0.001$ & $0.04 \pm 0.00$ & 552.95 & 1.05 & $1.04-1.05$ & $<0.001$ \\
\hline
\end{tabular}

The Backward Wald method was used for selecting the covariates in the multivariable analyses. $-2 \log$ likelihood $=4,955.53$

$S E$ standard error, $H R$ hazard ratio, $C I$ confidence intervals, $L T$ liver transplantation, $B M I$ body mass index, $M E L D$ model for end-stage liver disease, $C V A$ cerebrovascular accident, $I C U$ intensive care unit, DM diabetes mellitus, $C I T$ cold ischemia time, WIT warm ischemia time, BAR balance of risk, $D-M E L D$ donor-MELD, $E A D$ early allograft dysfunction, $C C I$ comprehensive complication index

${ }^{a}$ Variables initially introduced in the multivariable model

bThe multivariable model was constructed introducing only the variable "D-MELD", with the intent to avoid collinearity phenomena with the variable "MELD". A similar model with the variable "MELD" instead of "D-MELD" was contextually constructed. In both cases, D-MELD or MELD were deleted during the backward Wald method 
Table 4 Prediction of 90-day graft loss in the training and validation sets

\begin{tabular}{|c|c|c|c|c|c|c|c|}
\hline \multirow[t]{2}{*}{ Scores } & \multicolumn{3}{|c|}{ Training set $(N=1262)$} & \multirow[t]{2}{*}{ Scores } & \multicolumn{3}{|c|}{ Validation set $(N=520)$} \\
\hline & $\mathrm{AUC} \pm \mathrm{SE}$ & $95 \% \mathrm{CIs}$ & $p$ value & & $\mathrm{AUC} \pm \mathrm{SE}$ & $95 \%$ CIs & $p$ value \\
\hline $\mathrm{CCI}$ & $0.94 \pm 0.01$ & $0.92-0.96$ & $<0.001$ & $\mathrm{CCI}$ & $0.77 \pm 0.08$ & $0.62-0.93$ & $<0.001$ \\
\hline D-MELD & $0.60 \pm 0.02$ & $0.56-0.65$ & $<0.001$ & BAR & $0.57 \pm 0.06$ & $0.45-0.68$ & 0.36 \\
\hline MELD & $0.60 \pm 0.02$ & $0.56-0.65$ & $<0.001$ & EAD & $0.57 \pm 0.07$ & $0.43-0.71$ & 0.35 \\
\hline BAR & $0.60 \pm 0.02$ & $0.55-0.64$ & $<0.001$ & D-MELD & $0.56 \pm 0.08$ & $0.41-0.70$ & 0.43 \\
\hline EAD & $0.58 \pm 0.02$ & $0.53-0.62$ & 0.001 & MELD & $0.47 \pm 0.07$ & $0.33-0.61$ & 0.70 \\
\hline CCI cut-off & Sens & Spec & DOR & CCI cut-off & Sens & Spec & DOR \\
\hline 12.2 (25th) & 98.4 & 27.7 & 23.6 & 12.2 & 82.4 & 32.1 & 2.2 \\
\hline 29.6 (50th) & 96.8 & 56.1 & 38.7 & 29.6 & 76.5 & 52.1 & 3.5 \\
\hline 47.3 (75th) & 88.8 & 85.9 & 48.3 & 47.3 & 64.7 & 79.3 & 7.0 \\
\hline 84.9 (90th) & 66.3 & 98.7 & 149.4 & 84.9 & 41.2 & 98.6 & 49.3 \\
\hline
\end{tabular}

$A U C$ area under the curve, $S E$ standard error, $C I s$ confidence intervals, $C C I$ comprehensive complication index, $D$ - $M E L D$ donor-model for endstage liver disease, $M E L D$ model for end-stage liver disease, $E A D$ early allograft dysfunction, $B A R$ balance of risk, $D O R$ diagnostic odds ratio

corresponding to the median point (29.6) had a sensitivity of 96.8 and a specificity of $56.1(\mathrm{DOR}=38.7)$. The value of 47.3 , corresponding to the third quartile, exhibited a sensitivity of 88.8 and a specificity of 85.9 , giving a high DOR value of 48.3. Lastly, the threshold value put at 84.9 (ninth decile) showed a sensitivity $=66.3$ and a specificity $=98.7$ $(\mathrm{DOR}=149.4)($ Table 4$)$.

The same cut-offs validated in the validation set showed similar excellent diagnostic ability, although they were inferior in terms of discriminative power.

The CCI cut-off value at 12.2 (first quartile) yielded a sensitivity of 82.4 and a specificity of $32.1(\mathrm{DOR}=2.2)$. The cut-off set at 29.6 (median) had a sensitivity of 76.5 and a specificity of 52.1 (DOR $=3.5$ ). The cut-off put at 47.3 (third quartile) exhibited a sensitivity of 64.7 and a specificity of
79.3, giving a DOR value of 7.0. Lastly, the threshold value put at 84.9 (ninth decile) presented a sensitivity $=41.3$ and a specificity $=98.6(\mathrm{DOR}=49.3)($ Table 4$)$.

\section{1-year graft loss diagnostic ability}

The diagnostic ability of five different scoring systems was evaluated in both the sets, with the intent to identify the best diagnostic test for 1-year graft loss (Table 5).

CCI exhibited the best diagnostic performances in both training (AUC $=0.88,95 \% \mathrm{CI}=0.85-0.90 ; p<0.001)$ and validation sets (AUC $=0.75,95 \% \mathrm{CI}=0.65-0.85 ; p<0.001$ ). All the other scores always showed inferior AUCs, only ranging $0.56-0.59$ and $0.45-0.63$, respectively.

Table 5 Prediction of 1-year graft loss in the training and validation sets

\begin{tabular}{|c|c|c|c|c|c|c|c|}
\hline \multirow[t]{2}{*}{ Scores } & \multicolumn{3}{|c|}{ Training set $(N=1262)$} & \multirow[t]{2}{*}{ Scores } & \multicolumn{3}{|c|}{ Validation set $(N=520)$} \\
\hline & $\mathrm{AUC} \pm \mathrm{SE}$ & $95 \%$ CIs & $p$ value & & $\mathrm{AUC} \pm \mathrm{SE}$ & $95 \%$ CIs & $p$ value \\
\hline $\mathrm{CCI}$ & $0.88 \pm 0.02$ & $0.85-0.90$ & $<0.001$ & $\mathrm{CCI}$ & $0.75 \pm 0.05$ & $0.65-0.85$ & $<0.001$ \\
\hline D-MELD & $0.59 \pm 0.02$ & $0.55-0.63$ & $<0.001$ & D-MELD & $0.63 \pm 0.05$ & $0.54-0.73$ & 0.007 \\
\hline MELD & $0.59 \pm 0.02$ & $0.55-0.63$ & $<0.001$ & MELD & $0.54 \pm 0.05$ & $0.45-0.63$ & 0.40 \\
\hline BAR & $0.59 \pm 0.02$ & $0.55-0.63$ & $<0.001$ & EAD & $0.53 \pm 0.05$ & $0.43-0.63$ & 0.50 \\
\hline$\underline{E A D}$ & $0.56 \pm 0.02$ & $0.52-0.60$ & 0.004 & BAR & $0.45 \pm 0.05$ & $0.36-0.55$ & 0.32 \\
\hline CCI cut-off & Sens & Spec & DOR & CCI cut-off & Sens & Spec & DOR \\
\hline 12.2 (25th) & 94.9 & 28.6 & 7.5 & 12.2 & 86.5 & 33.1 & 3.2 \\
\hline 29.6 (50th) & 89.1 & 57.8 & 11.2 & 29.6 & 78.4 & 53.4 & 4.2 \\
\hline 47.3 (75th) & 75.0 & 87.5 & 21.0 & 47.3 & 56.8 & 80.5 & 5.4 \\
\hline 84.9 (90th) & 53.5 & 99.9 & $1,149.4$ & 84.9 & 27.0 & 99.2 & 45.9 \\
\hline
\end{tabular}

$A U C$ area under the curve, $S E$ standard error, $C I S$ confidence intervals, $C C I$ comprehensive complication index, $D$ - $M E L D$ donor-model for endstage liver disease, $M E L D$ model for end-stage liver disease, $E A D$ early allograft dysfunction, $B A R$ balance of risk, $D O R$ diagnostic odds ratio 
In the training set, the CCI cut-off value corresponding to the first quartile (12.2 points) yielded a sensitivity of 94.9 and a specificity of $28.6(\mathrm{DOR}=7.5)$. The threshold value corresponding to the median point (29.6) had a sensitivity of 89.1 and a specificity of $57.8(\mathrm{DOR}=11.2)$. The value of 47.3 , corresponding to the third quartile, exhibited a sensitivity of 75.0 and a specificity of 87.5 , giving a high DOR value of 21.0. Lastly, the threshold value put at 84.9 (ninth decile) showed a sensitivity $=53.5$ and a specificity $=99.9$ $(\mathrm{DOR}=1149.4)$ (Table 5).

The same cut-offs validated in the validation set showed similar excellent diagnostic ability, although they were inferior in terms of discriminative power.

The CCI cut-off value at 12.2 (first quartile) yielded a sensitivity of 86.5 and a specificity of $33.1(\mathrm{DOR}=3.2$ ). The cut-off set at 29.6 (median) had a sensitivity of 78.4 and a specificity of $53.4(\mathrm{DOR}=4.2)$. The cut-off put at 47.3 (third quartile) exhibited a sensitivity of 56.8 and a specificity of 80.5 , giving a DOR value of 5.4. Lastly, the threshold value put at 84.9 (ninth decile) presented a sensitivity $=27.0$ and a specificity $=99.2(\mathrm{DOR}=45.9)($ Table 5$)$.

\section{Sub-analysis on the graft loss diagnostic ability using aged grafts}

The diagnostic ability of the five different scoring systems was also evaluated in a sub-analysis in which only transplants performed using organs from aged ( $\geq 70$ years) donors were considered (Table 6). As for the 90-day risk of graft loss, CCI confirmed the best diagnostic performances in both training $(\mathrm{AUC}=0.93,95 \% \mathrm{CI}=0.88-0.97 ; p<0.001$ ) and validation sets $(\mathrm{AUC}=0.92,95 \% \mathrm{CI}=0.81-1.00$; $p=0.001$ ). Similarly, CCI was also the best diagnostic tool for predicting 1-year graft loss, with the best diagnostic performances in both training (AUC $=0.88,95 \%$ $\mathrm{CI}=0.82-0.93 ; p<0.001)$ and validation sets $(\mathrm{AUC}=0.79$, $95 \% \mathrm{CI}=0.59-1.00 ; p=0.002)$.

\section{Graft survival rates}

In the training set, we obtained an excellent stratification of graft survival rates using the investigated CCI thresholds.

For instance, 1-year graft survival rates were 94.7, 95.3, 88.5, 68.7, and $0.7 \%$ in patients with CCI $0.0-12.2$, 12.3-29.6, 29.7-47.3, 47.4-84.9, and 85.0-100.0, respectively (log-rank $p<0.001)$ (Fig. 1a).

In the validation set, we similarly obtained a good stratification of graft survivals. For instance, 1-year graft survival rates were $96.0,99.2,93.3,88.9$, and $23.1 \%$ in patients with CCI 0.0-12.2, 12.3-29.6, 29.7-47.3, 47.4-84.9, and $85.0-100.0$, respectively (log-rank $p<0.001)$ (Fig. 1b).

\section{Discussion}

A valid scoring system should exhibit good performance metrics, such as discrimination and calibration, to maintain these qualities over time, and it should be simple and easy to calculate.

In the specific setting of LT, MELD score covers most of these characteristics when used for the prediction of death during the waiting time. This ability was the reason for the introduction of the MELD score, in 2002, in the US liver allocation process. The goal was to prioritize the sickest patients for transplantation [14]. However, the MELD score rapidly proved to be a poor predictor of short- and, worse,
Table 6 Prediction of 90-day and 1-year graft loss in the training and validation sets: transplants performed using organs from donors with age $\geq 70$ years

\begin{tabular}{|c|c|c|c|c|c|c|c|}
\hline \multirow[t]{2}{*}{ Scores } & \multicolumn{3}{|c|}{ Training set $(N=1262)$} & \multirow[t]{2}{*}{ Scores } & \multicolumn{3}{|c|}{ Validation set $(N=520)$} \\
\hline & $\mathrm{AUC} \pm \mathrm{SE}$ & $95 \% \mathrm{CIs}$ & $p$ value & & $\mathrm{AUC} \pm \mathrm{SE}$ & $95 \% \mathrm{CIs}$ & $p$ value \\
\hline \multicolumn{8}{|c|}{ 90-Day graft loss } \\
\hline CCI & $0.93 \pm 0.02$ & $0.88-0.97$ & $<0.001$ & $\mathrm{CCI}$ & $0.92 \pm 0.06$ & $0.81-1.00$ & 0.001 \\
\hline BAR & $0.59 \pm 0.04$ & $0.52-0.67$ & 0.03 & BAR & $0.55 \pm 0.11$ & $0.34-0.76$ & 0.69 \\
\hline EAD & $0.53 \pm 0.04$ & $0.45-0.61$ & 0.50 & D-MELD & $0.53 \pm 0.12$ & $0.30-0.77$ & 0.80 \\
\hline MELD & $0.53 \pm 0.05$ & $0.44-0.61$ & 0.52 & EAD & $0.50 \pm 0.12$ & $0.26-0.74$ & 0.99 \\
\hline D-MELD & $0.52 \pm 0.05$ & $0.43-0.61$ & 0.64 & MELD & $0.46 \pm 0.11$ & $0.26-0.68$ & 0.77 \\
\hline \multicolumn{8}{|c|}{ 1-year graft loss } \\
\hline CCI & $0.88 \pm 0.03$ & $0.82-0.93$ & $<0.001$ & $\mathrm{CCI}$ & $0.79 \pm 0.10$ & $0.59-1.00$ & 0.002 \\
\hline BAR & $0.57 \pm 0.04$ & $0.50-0.64$ & 0.06 & BAR & $0.53 \pm 0.10$ & $0.34-0.71$ & 0.78 \\
\hline EAD & $0.53 \pm 0.04$ & $0.46-0.60$ & 0.49 & EAD & $0.52 \pm 0.09$ & $0.33-0.70$ & 0.87 \\
\hline D-MELD & $0.52 \pm 0.04$ & $0.44-0.59$ & 0.69 & D-MELD & $0.49 \pm 0.11$ & $0.28-0.70$ & 0.95 \\
\hline MELD & $0.52 \pm 0.04$ & $0.45-0.60$ & 0.51 & MELD & $0.47 \pm 0.10$ & $0.27-0.66$ & 0.71 \\
\hline
\end{tabular}

$A U C$ area under the curve, $S E$ standard error, $C I S$ confidence intervals, $C C I$ comprehensive complication index, $D$-MELD donor-model for end-stage liver disease, $M E L D$ model for end-stage liver disease, $E A D$ early allograft dysfunction, $B A R$ balance of risk, $D O R$ diagnostic odds ratio 


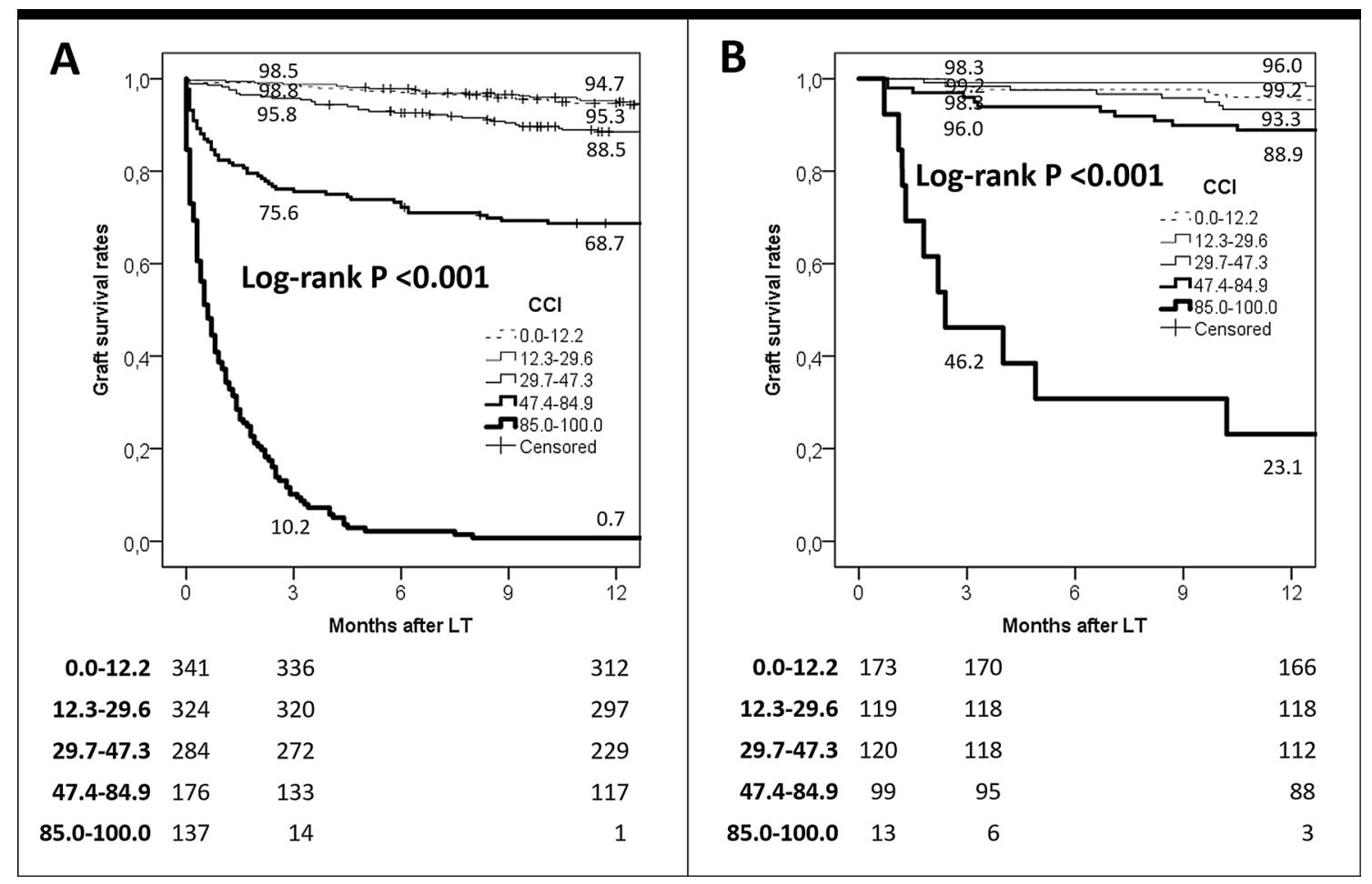

Fig. 1 a Training set: 1-year graft survival rates according to the CCI risk strata. b Validation set: 1-year graft survival rates according to the CCI risk strata

long-term post-transplant survival [15-17]. A recent systematic review, which included 37 studies covering 53,691 patients transplanted in 15 different countries, identified an overall c-statistics inferior to 0.7 and consequently suggested a global poor predictive value of the score [4].

With the intent to improve its predictive ability, the MELD score has been integrated into different models built to enhance the prediction of post-transplant survival. Unfortunately, the complexity of many of these models limits their usability. The Survival Outcomes Following Liver Transplantation (SOFT) score represents a paradigmatic example: despite its good predictive ability, the score is based on 18 different pre-transplant variables, making it difficult to calculate [18]. The same holds for the MELD-sarcopenia score, in which the single complex-to-estimate parameter "sarcopenia" limits its broad applicability [19]. Conversely, the D-MELD, based on the simple multiplication of donor age and recipient MELD, represents an easy-to-calculate model $[5,20]$. The BAR score, based on six donor- and recipientrelated pre-operative variables, further improves prognostication without excessively increasing the complexity [6]. Moreover, a web calculator is available for its estimation. The BAR score has proven to offer great potential in different geographical areas [6, 21, 22]. A Chinese study including 249 LDLT patients showed that the BAR score was the best predictor of 1-year patient survival [21]. A Brazilian study including 402 patients reported similar results when looking at three-month patient survival [22].

However, all these scores based on pre-transplant data typically yielded inferior results compared to scoring systems based on variables available in the immediate posttransplant period. Among the post-transplant scores, the Olthoff-EAD is the most commonly adopted [7, 23].

The great and largely unsolved challenge in LT remains how to correctly allocate a limited resource such as organs from deceased donors, which can be addressed only with preoperative variables. Therefore, a score composed by post-transplant parameters cannot be used with the intent to optimize the allocation process. However, such a score should maintain its potential usefulness as a diagnostic tool for early (i.e., 3-month, 1-year) clinical course prediction.

In the present series, we observed that the CCI model presented high relevance for LT survival prognostication in both the Sets we investigated. Moreover, CCI outperformed both the pre- and post-transplant scores in diagnostic ability.

CCI was initially created to report complication rates more accurately. The CCI aimed to inform about the severity of cumulative postoperative complications precisely [8]. Recently, its potential role as a prognostic tool has been implemented in different fields of surgery. As an example, two international studies used CCI cut-off values of 33 and 42 as benchmarks for evaluating the quality of a 
successfully performed liver resection or transplantation, respectively [10, 24].

Several studies investigated the prognostic impact of $\mathrm{CCI}$ in the setting of different types of cancer. A US study showed that CCI was a strong survival predictor in patients undergoing hepatic resection for colorectal metastases independently from the RAS mutational status. Patients with high CCI $(\geq 26.2)$ had worse recurrence-free and cancer-specific survivals with respect to low-CCI patients [9].

A study from Japan correlated postoperative complications with worse survivals in gastric cancer patients. Patients with a CCI $\geq 32.15$ had significantly lower 5-year overall and disease-specific survivals than those observed in the CCI low group. Moreover, a multivariate analysis identified the CCI as an independent prognostic indicator [25]. Another study from China similarly investigated the role of CCI in the setting of gastric cancer. Patients with high CCI $(\geq 26.2)$ presented 5-year cancer-specific survival rates markedly inferior ( $46.3 \%$ vs. $54.9 \%$ ) [26].

CCI was also correlated with several parameters of poor outcome after surgery, further explaining its potential role as a predictor of poor early outcomes. A study from Spain correlated CCI with the frailty status in elderly patients treated with surgery, suggesting a correlation among frailness, post-surgical complications, and poor outcomes [27]. Another US study showed a correlation between CCI and time to normal activity in patients undergoing gastrointestinal and hepato-bilio-pancreatic surgery [28].

Up to now, only one Dutch study has revealed a prognostic role of CCI in the specific setting of liver transplantation. Specifically, when transplants performed with organs from deceased-cardiac donors (DCD) or deceasedbrain donors (DBD) were compared, 6-month postoperative median CCI was significantly higher in case of DCD grafts (53.4 vs 47.2). Moreover, more DCD recipients underwent re-transplantation for ischemic-type biliary lesions in this period (4\% vs $1 \%$ ), therefore suggesting a correlation between CCI and the development of biliary complications [11].

In the present experience, the CCI reported the best diagnostic ability respect to all the other tested scores in terms of graft loss risk, with AUCs of 0.94 and 0.77 in the training and validation sets for the diagnosis of 90-day graft loss. As for 1-year graft loss, CCI showed similar good performances in the training $(\mathrm{AUC}=0.88)$ and validation sets $(\mathrm{AUC}=0.75)$.

The strength of the CCI was particularly evident in light of the poor performances observed by the other tested preand post-transplant scores. Interestingly, no one of them ever showed an AUC $>0.70$ in both the 90-day and 1-year graft loss risk estimation.

We also tested several CCI cut-offs. Interestingly, the value corresponding to the third quartile (47.3) was substantially similar to the threshold identified by Muller et al. on 7492 patients transplanted in 17 different centers [24].

We clearly understand that the diagnostic utility of CCI should appear marginal, mainly in consideration of the potentially long time required for its calculation. Typically, scores based on post-transplant data are collected within seven to ten days from LT [7, 23]. While the CCI calculation was set in our study at the time of patient discharge. However, just for clarifying the timeframes required for the CCI estimation, 1067/1262 (84.5\%) and 464/520 (89.2\%) patients were discharged in the training and validation sets within one month from LT, thus consenting to obtain the CCI calculation in an acceptable time, mainly in light of its usefulness for the prediction of 1-year graft loss.

Another aspect to consider is the fact that, once a LT patient has developed a complication, the ability to improve the patient outcomes should be markedly limited if compared with the possibility to pre-operatively prevent this specific complication. We understand this shortcoming of the model, obviously limiting the impact on the CCI in conditioning important aspects like an early re-transplantation. However, we think the role of the CCI merits consideration, mainly in light of the possibility to identify patients that are more "fragile" at the discharge time.

As an example, the sub-analysis focused on the transplants performed using aged grafts showed that the CCI even improved its diagnostic ability to predict early graft loss, therefore underlying the potential utility of this score in identifying transplanted patients at time of discharge requiring particular attentions during the follow-up.

As previously reported, the CCI should play a role in the prognosis of tumoral patients [10, 25, 26]. Studies on colorectal metastases and gastric cancer have been reported $[10,25,26]$, while no studies have been published up to now with the intent to correlate CCI and post-transplant HCC recurrence risk. The present study was not constructed with the aim of investigating the correlation between HCC, LT and CCI. However, we can postulate that, also in this case, a worse correlation between high CCI and cancer should exist. Therefore, in tumor patients with high CCI at time of discharge, we should justify the use of a tailored immunosuppression (i.e., everolimus, rapid steroid withdrawal), a more cautious use of steroid boluses in the management of acute rejections, or a personalized scheme of outpatient follow-up (i.e., more frequent measurements of alpha-fetoprotein or a more stringent imaging protocol). Further studies specifically focused on the correlation between CCI and HCC are required.

Another important element is the potential correlation between CCI and biliary complications. Only one study specifically reported this connection, therefore requiring more detailed studies with the intent to clarify the potential 
intercorrelation between poor initial clinical course and biliary complications [9]. However, also in this case, we can postulate that the early identification of patients with a greater risk for biliary complications should offer the opportunity to design tailored therapies (i.e., ursodesoxycholic acid) and personalized schemes of outpatient followup comprehending early magnetic resonance imaging, with the intent to minimize possible complications.

We think such an opportunity is not of marginal relevance. As an example, 176/1262 (13.9\%) and 102/520 $(19.6 \%)$ patients in the training and validation sets overpassing the identified threshold of 47.3 were alive at discharging time. In the training set, $67 / 102(38.1 \%)$ of these patients had biliary complications, and $40(22.7 \%)$ required a retransplantation during their follow-up. We are confident that these patients should potentially benefit from a modification of the post-discharge management policy, due to the peculiar condition derived from a complex post-transplant course.

One can argue that a potential bias of the study is represented by the arbitrary decision to calculate the CCI only at the time of the first post-LT hospitalization, excluding the possible complications observed by the patient after discharge. As an example, in the study by Muller et al., the CCI value was calculated at 12 months after transplantation [24]. On the opposite, we voluntarily decided to measure the $\mathrm{CCI}$ at the time of discharge. In fact, we think that such a measurement gives the opportunity to identify a sub-class of high-risk cases at discharge in which the previously reported management changes should be adopted with the intent to minimize their predictable poorer clinical course.

The intent of the study was not to compare the training and the validation sets. However, in light of the observed results, we noted that the Validation Set reported better CCI and 90-day results despite a higher median MELD value. We can do some suppositions for explaining this paradoxical result. First, MELD alone is not necessarily able to capture the overall recipient technical difficulties, mainly in case of "exception" pathologies like HCC and biliary cholangiopathies. In approximately half of the Training and Validation Set cases, we exactly observed these types of pathologies, respectively. Second, the high number of HCC cases in the training set should explain the higher rate of vascular thromboses/stenoses as a consequence of several intra-arterial treatments caused by bridging/downstaging strategies $[29,30]$. Third, a possible effect of institutional case volume should explain this result. The Validation Set is, in fact, a high-volume center, representing a centralized referral and management center for all the hepatopathies of its country. Several studies already reported better results in high- vs. mediumvolume centers $[31,32]$. Another aspect to consider is the higher percentage of PNF observed in the training set (2.9 vs. $0.6 \%, p=0.001$ ), potentially explainable with worse histological aspects of the used graft. Unfortunately, due to the retrospective nature of the study, we were not able to explore this aspect. Last, we cannot exclude the presence of comorbidities like refractory ascites and portal hypertension in the recipients potentially justifying the observed results. Also in this case, we were not able to retrospectively evaluate in detail these aspects.

We are aware that the study may have some limitations. First, the study is retrospective and pluricentric. The main concern connected with the retrospective nature of the study is the risk of missed post-LT complications, mainly for the grade I-II cases. A systematic retrospective collection of all the pharmacological needs of LT patients should be challenging. However, we can say that, although potentially underestimated, the diagnostic effect of CCI was particularly relevant in our series. Consequently, we can only assert that the CCI role should be even stronger in a prospectively collected database.

Another limit of the study is connected with the fact that some complications have been classified for CCI scoring on a non-empirical basis, due to a lack of literature investigating on this aspect. Obviously, such a condition is of relevance because the heterogeneity in the CCI grading of specific complications may influence the performance characteristics of the score.

As for the multicentricity of the training set, we should emphasize that the large sample size of this population should minimize possible biases related to data analysis. Moreover, the validation set was based only on a monocentric experience, however confirming an overall broad prognostic ability of CCI also in this context.

In conclusion, the CCI shows a very good diagnostic ability for 90-day and 1-year graft loss in both a multicentric training and a monocentric validation set. Its diagnostic power is superior to other commonly adopted pre- and post-LT scores. Further analyses are required to prove its validity even in the long term.

Acknowledgements The authors thank Marta Fiorenza for her kind linguistic revision. Collaborators: Zoe Larghi Laureiro (General Surgery and Organ Transplantation Unit, Department of Surgery, Sapienza University of Rome, Umberto I Polyclinic of Rome, Rome, Italy Sapienza Rome); Susanna Mazzoccato (Unit of Hepatobiliary Surgery and Transplantation, Polytechnic University of Marche, Azienda Ospedaliero-Universitaria "Ospedali Riuniti” Torrette, Ancona, Italy); Maxime Foguenne and Tiziana Fabbrizio (Starzl Unit of Abdominal Transplantation, Pôle de Chirurgie Expérimentale et Transplantation, Institut de Recherche Expérimentale et Clinique, Université catholique de Louvain, Brussels, Belgium); Alessandro Vitale (Department of Surgery, Oncology and Gastroenterology, University of Padua, Padua, Italy).

Author contributions QL, FMe, and JL participated in research design; QL participated in data analysis; QL, SI, and JL participated in the writing of the paper; FMe, GN, DN, SI, EF, GM, AR, FMo, KA, MP, LM OC, GZ, MV, UC, MR, and BGE participated in the performance 
of the research; All the Authors gave final approval for the paper to be published.

Funding Open access funding provided by Università degli Studi di Roma La Sapienza within the CRUI-CARE Agreement.

\section{Compliance with ethical standards}

Conflict of interest The authors declare no conflicts of interest.

Research involving human participants and/or animals A study-specific approval was obtained by the local ethical committee of Sapienza University of Rome Policlinico Umberto I of Rome (leading center of the study).

Informed consent The Authors obtained an informed consent at the time of transplantation from all the participants of the study for the treatment of their clinical data.

Open Access This article is licensed under a Creative Commons Attribution 4.0 International License, which permits use, sharing, adaptation, distribution and reproduction in any medium or format, as long as you give appropriate credit to the original author(s) and the source, provide a link to the Creative Commons licence, and indicate if changes were made. The images or other third party material in this article are included in the article's Creative Commons licence, unless indicated otherwise in a credit line to the material. If material is not included in the article's Creative Commons licence and your intended use is not permitted by statutory regulation or exceeds the permitted use, you will need to obtain permission directly from the copyright holder. To view a copy of this licence, visit http://creativecommons.org/licenses/by/4.0/.

\section{References}

1. Kamath PS, Wiesner RH, Malinchoc M et al (2001) A model to predict survival in patients with end-stage liver disease. Hepatology 33:464-470

2. Freeman RB (2012) A decade of model for end-stage liver disease: lessons learned and need for re-evaluation of allocation policies. Curr Opin Organ Transplant 17:211-215

3. Silberhumer GR, Hetz H, Rasoul-Rockenschaub S et al (2006) Is MELD score sufficient to predict not only death on waiting list, but also post-transplant survival? Transplant Int 19:275-281

4. Klein KB, Stafinski TD, Menon D (2013) Predicting survival after liver transplantation based on pre-transplant MELD score: a systematic review of the literature. PLoS ONE 8:e80661

5. Halldorson JB, Bakthavatsalam R, Fix O et al (2009) D-MELD, a simple predictor of post liver transplant mortality for optimization of donor/recipient matching. Am J Transplant 9:318-326

6. Dutkowski P, Oberkofler CE, Slankamenac K et al (2011) Are there better guidelines for allocation in liver transplantation? A novel score targeting justice and utility in the model for end-stage liver disease era. Ann Surg 254:745-753

7. Olthoff KM, Kulik L, Samstein B et al (2010) Validation of a current definition of early allograft dysfunction in liver transplant recipients and analysis of risk factors. Liver Transplant 16:943-949

8. Slankamenac K, Graf R, Barkun J et al (2013) The comprehensive complication index: a novel continuous scale to measure surgical morbidity. Ann Surg 258:1-7

9. Yamashita S, Sheth RA, Niekamp AS et al (2017) Comprehensive complication index predicts cancer-specific survival after resection of colorectal metastases independent of RAS mutational status. Ann Surg 266:1045-1054

10. Rössler F, Sapisochin G, Song G et al (2016) Defining benchmarks for major liver surgery: a multicentre analysis of 5202 living liver donors. Ann Surg 264:492-500

11. Kalisvaart M, de Haan JE, Polak WG et al (2017) Comparison of postoperative outcomes between donation after circulatory death and donation after brain death liver transplantation using the comprehensive complication index. Ann Surg 266:772-778

12. Dindo D, Demartines N, Clavien PA (2004) Classification of surgical complications: a new proposal with evaluation in a cohort of 6336 patients and results of a survey. Ann Surg 240:205-213

13. Sainani KL (2013) Multivariate regression: the pitfalls of automated variable selection. PM R 5:791-794

14. Freeman RB Jr, Wiesner RH, Harper A, UNOS/OPTN Liver Disease Severity Score, UNOS/OPTN Liver, and Intestine, and UNOS/ OPTN Pediatric Transplantation Committees et al (2002) The new liver allocation system: moving toward evidence-based transplantation policy. Liver Transplant 8:851-858

15. Duan BW, Lu SC, Wu JS et al (2014) Model for end-stage liver disease (MELD) score does not predict outcomes of hepatitis $\mathrm{B}$-induced acute-on-chronic liver failure in transplant recipients. Transplant Proc 46:3502-3506

16. Yadav SK, Saraf N, Saigal S et al (2017) High MELD score does not adversely affect outcome of living donor liver transplantation: experience in 1000 recipients. Clin Transplant 31:e13006

17. Weismüller TJ, Fikatas P, Schmidt J et al (2011) Multicentric evaluation of model for end-stage liver disease-based allocation and survival after liver transplantation in Germany-limitations of the 'sickest first'-concept. Transplant Int 24:91-99

18. Rana A, Hardy MA, Halazun KJ et al (2008) Survival outcomes following liver transplantation (SOFT) score: a novel method to predict patient survival following liver transplantation. Am J Transplant 8:2537-2546

19. Montano-Loza AJ, Duarte-Rojo A, Meza-Junco J et al (2015) Inclusion of sarcopenia within MELD (MELD-Sarcopenia) and the prediction of mortality in patients with cirrhosis. Clin Transl Gastroenterol 6:e102

20. Avolio AW, Cillo U, Salizzoni M, Donor-to-Recipient Italian Liver Transplant (D2R-ILTx) Study Group et al (2011) Balancing donor and recipient risk factors in liver transplantation: the value of D-MELD with particular reference to HCV recipients. Am J Transplant 11:2724-2736

21. Ma Y, Wang Q, Yang J et al (2015) Comparison of different scoring systems based on both donor and recipient characteristics for predicting outcome after living donor liver transplantation. PLoS ONE 10:e0136604

22. de Campos Junior ID, Stucchi RS, Udo EY et al (2015) Application of the BAR score as a predictor of short- and long-term survival in liver transplantation patients. Hepatol Int 9:113-119

23. Pareja E, Cortes M, Hervás D et al (2015) A score model for the continuous grading of early allograft dysfunction severity. Liver Transplant 21:38-46

24. Muller X, Marcon F, Sapisochin G et al (2018) Defining benchmarks in liver transplantation: a multicentre outcome analysis determining best achievable results. Ann Surg 267:419-425

25. Shimizu S, Saito H, Kono Y et al (2019) The prognostic significance of the comprehensive complication index in patients with gastric cancer. Surg Today 49:913-920

26. Tu RH, Lin JX, Li P et al (2018) Comprehensive complication index predicts cancer-specific survival of patients with postoperative complications after curative resection of gastric cancer. Gastroenterol Res Pract 2018:4396018 
27. Artiles-Armas M, Roque-Castellano C, Conde-Martel A, MarchenaGómez J (2019) The comprehensive complication index is related to frailty in elderly surgical patients. J Surg Res 244:218-224

28. Ray S, Mehta NN, Mangla V et al (2019) A comparison between the comprehensive complication index and the Clavien-Dindo grading as a measure of postoperative outcome in patients undergoing gastrointestinal surgery-a prospective study. J Surg Res 244:417-424

29. Goel A, Mehta N, Guy J et al (2014) Hepatic artery and biliary complications in liver transplant recipients undergoing pretransplant transarterial chemoembolization. Liver Transplant 20:1221-1228

30. Sneiders D, Houwen T, Pengel LHM et al (2018) Systematic review and meta-analysis of posttransplant hepatic artery and biliary complications in patients treated with transarterial chemoembolization before liver transplantation. Transplantation 102:88-96
31. Yoo S, Jang EJ, Yi NJ et al (2019) Effect of institutional case volume on in-hospital mortality after living donor liver transplantation: analysis of 7073 cases between 2007 and 2016 in Korea. Transplantation 103:952-958

32. Ozhathil DK, Li YF, Smith JK et al (2011) Impact of center volume on outcomes of increased-risk liver transplants. Liver Transplant 17:1191-1199

Publisher's Note Springer Nature remains neutral with regard to jurisdictional claims in published maps and institutional affiliations. 